\title{
Surgical video recording with a modified GoPro Hero 4 camera
}

This article was published in the following Dove Press journal:

Clinical Ophthalmology

13 January 2016

Number of times this article has been viewed

\section{Lily Koo Lin}

Department of Ophthalmology and Vision Science, University of California, Davis Eye Center, Sacramento, CA, USA
Correspondence: Lily Koo Lin University of California, Davis Eye Center, 4860 Y Street, Suite 2400, Sacramento, CA 958I7, USA

$\mathrm{Tel}+\mathrm{I} 9167346602$

Fax + I 91673404||

Email Iklin@ucdavis.edu
Background: Surgical videography can provide analytical self-examination for the surgeon, teaching opportunities for trainees, and allow for surgical case presentations. This study examined if a modified GoPro Hero 4 camera with a $25 \mathrm{~mm}$ lens could prove to be a cost-effective method of surgical videography with enough detail for oculoplastic and strabismus surgery.

Method: The stock lens mount and lens were removed from a GoPro Hero 4 camera, and was refitted with a Peau Productions SuperMount and $25 \mathrm{~mm}$ lens. The modified GoPro Hero 4 camera was then fixed to an overhead surgical light.

Results: Camera settings were set to $1080 \mathrm{p}$ video resolution. The $25 \mathrm{~mm}$ lens allowed for nine times the magnification as the GoPro stock lens. There was no noticeable video distortion. The entire cost was less than 600 USD.

Conclusion: The adapted GoPro Hero 4 with a $25 \mathrm{~mm}$ lens allows for high-definition, costeffective, portable video capture of oculoplastic and strabismus surgery. The $25 \mathrm{~mm}$ lens allows for detailed videography that can enhance surgical teaching and self-examination.

Keywords: teaching, oculoplastic, strabismus

\section{Introduction}

There is no question that reviewing surgical video recordings can provide analytical self-reflection for the surgeon and teaching opportunities for surgical trainees, and allow for surgical case presentations to a wider audience at conferences and meetings. This has been a long-held standard for ocular surgeons who utilize the surgical microscope; however, recording surgery has been relatively elusive for the oculoplastic or strabismus surgeon. Often operating room video systems can be costly, and for many hospitals and practices, have been a limiting factor in being able to record non-microscopic ophthalmic surgery. The author presents a modified, commercially available, GoPro Hero 4 camera as a means to provide high-definition surgical recordings.

\section{Technique}

A GoPro Hero 4 camera (GoPro, Inc., San Mateo, CA, USA) was refitted with a Peau Productions SuperMount (Peau Productions, Inc., San Diego, CA, USA). The SuperMount is a fabricated lens mount that accommodates alternate lenses. The GoPro stock lens and lens mount were removed, and the Peau Productions SuperMount was installed. No cutting, gluing, or breaking of components was required for installing the SuperMount, as the GoPro stock lens and lens mount are removed together as one piece. The installation of the SuperMount video is available for viewing on www. peauproductions.com. The GoPro camera with an already installed SuperMount is also an option for purchase on the website. 
Once the SuperMount was placed, a $25 \mathrm{~mm}$ lens (Peau Productions, Inc., \$80) was placed inside the SuperMount. The GoPro Hero 4 camera was placed in an open-frame mount rather than the stock waterproof housing (GoPro Frame mount by CamKix, \$19.99) to allow for ease of focusing the $25 \mathrm{~mm}$ lens. The GoPro camera can be fixed to an overhead surgical light with either a GoPro suction mount or a GoPro Jaws:Flex Clamp. A 15-feet HDMI cable with an HDMI to DVI adapter cable allowed for the image from the GoPro camera to be projected onto computer monitors already available in the operating room. This was done because the preview picture on the GoPro App was of lower resolution than the projected image on the high-resolution monitor and ensured that the video captured was in focus. The total cost of this system was less than 600 USD.

Due to the popularity of the GoPro camera and how the lens is mounted, the ability to both purchase and refit this particular camera with alternate lenses is readily available. The ability to refit or change lenses does not seem possible with other video cameras. Video recording was done at 1,080 p. Compared to the GoPro stock lens, which has a $128^{\circ}$ horizontal field of view, the $25 \mathrm{~mm}$ lens has a $14^{\circ}$ horizontal field of view. Field of view and magnification are inversely related, and thus, the $25 \mathrm{~mm}$ lens is roughly nine times the magnification over the GoPro stock lens. This viewing angle allows for excellent magnification while still allowing for the camera to be mounted on an overhead surgical light, away from the sterile field. There is minimal video image distortion, and there is no need for video image correction.

This system differs from a previously described GoPro camera modification from Maamari et al. ${ }^{1}$ Maamari et al ${ }^{1}$ described reconfiguring a GoPro Hero 3 camera using a $16 \mathrm{~mm}$ lens (RageCams, Inc., Sparta, MI, USA) posing the technical challenge of separating the GoPro stock lens from the GoPro stock mount, which are glued together. They also noted a video-recording artifact that needed to be corrected for with an optical density filter. Other published reports describe using a GoPro camera "out of the box" without any stock lens modification, which does not provide high enough magnification or resolution for oculoplastic or strabismus surgery. ${ }^{2-4}$

\section{Outcomes}

A still image from a video isolating the lateral rectus muscle during enucleation surgery is seen in Figure 1. The corresponding surgical video can be viewed in Video S1. Müllers muscle conjunctival resection ptosis repair surgery can be viewed in Video $\mathrm{S} 2$.

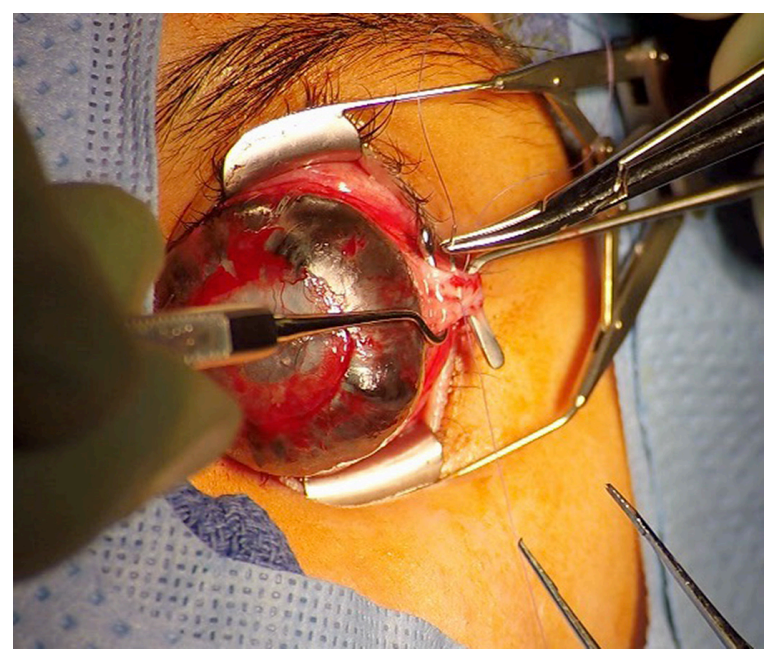

Figure I Still image capture from video recording of the isolated lateral rectus muscle during enucleation surgery.

\section{Discussion}

Given that the field of view is narrow, and no personal identifying factor of the patient was either linked or incorporated in the recording, and that surgical video recording is a normal educational practice, this study was exempt from Institutional Review Board approval of the University of California, Davis. Patients do sign a consent authorizing the use of any video or photography for training and teaching purposes including its use in lectures and medical journals. Given that the video is not linked to the patient's actual medical record, the video would not be available or known to prosecutors for medicolegal purposes.

Currently, our microsurgery colleagues, who have been routinely recording surgery on the surgical microscope for many years, do not require video proof of competency. Although there are some job positions that are requesting surgical videos, in truth, even the microsurgeons have no way to "prove" that the hands in the video do indeed belong to them, so it may be difficult to use this technology to "prove" competency.

Learning a surgical skill set is not unlike other skill sets. It requires countless hours of practice as well as critical self-examination. There are different aspects of learning that take place when one observes, and another when one is actively participating. There is certainly a role for both. Surgical videography can serve as an important retrospective and introspective supplemental learning experience for any surgeon; however, it cannot replace active participation.

\section{Conclusion}

The adapted GoPro Hero 4 camera with a $25 \mathrm{~mm}$ lens allows for easy, high-quality, cost-effective video capture 
of oculoplastic and strabismus surgery. The system is lightweight and portable, allowing for video recording in any operating room, minor room, and office. Furthermore, it enhances surgical teaching and self-examination.

\section{Disclosure}

The author reports no conflicts of interest in this work.

\section{References}

1. Maamari R, Vemuri S, Tao J. A modified action camera for high-quality, cost-effective oculofacial surgical videography. Ophthal Plast Reconstr Surg. 2015;31(4):336-337.
2. Paro JAM, Nazareli R, Gurjala A, Berger A, Lee GK. Video-based selfreview: comparing Google Glass and GoPro technologies. Ann Plast Surg. 2015;74(S1):S71-S73.

3. Bizzotto N, Sandri A, Lavini F, Dall'Oca C, Regis D. Video in operating room: GoPro HERO3 camera on surgeon's head to film operations- a test. Surg Innov. 2014;21(3):338-340.

4. Graves SN, Shenaq DS, Langerman AJ, Song DH. Video capture of plastic surgery procedures using the GoPro HERO3+. Plast Reconstr Surg Glob Open. 2015;3(2):e312.
Clinical Ophthalmology

\section{Publish your work in this journal}

Clinical Ophthalmology is an international, peer-reviewed journal covering all subspecialties within ophthalmology. Key topics include: Optometry; Visual science; Pharmacology and drug therapy in eye diseases; Basic Sciences; Primary and Secondary eye care; Patient Safety and Quality of Care Improvements. This journal is indexed on

Submit your manuscript here: http://www.dovepress.com/clinical-ophthalmology-journal

\section{Dovepress}

PubMed Central and CAS, and is the official journal of The Society of Clinical Ophthalmology (SCO). The manuscript management system is completely online and includes a very quick and fair peer-review system, which is all easy to use. Visit http://www.dovepress.com/ testimonials.php to read real quotes from published authors. 